\title{
Unveiling New Magnetic Phases of Undoped and Doped Manganites
}

\author{
Takashi Hotta ${ }^{1}$, Mohammad Moraghebi $^{2}$, Adrian Feiguin ${ }^{3}$, Adriana Moreo $^{2}$, Seiji Yunoki ${ }^{4}$, and Elbio Dagotto ${ }^{2}$ \\ ${ }^{1}$ Advanced Science Research Center, Japan Atomic Energy Research Institute, Tokai, Ibaraki 319-1195, Japan \\ ${ }^{2}$ National High Magnetic Field Laboratory, Florida State University, Tallahassee, Florida 32306 \\ ${ }^{3}$ Department of Physics and Astronomy, University of California at Irvine, CA 92697 \\ ${ }^{4}$ International School for Advanced Studies (SISSA), via Beirut 4, 34014 Trieste, Italy
}

(Dated: October 28, 2018)

\begin{abstract}
Novel ground-state spin structures in undoped and lightly-doped manganites are here investigated based on the orbital-degenerate double-exchange model, by using mean-field and numerical techniques. In undoped manganites, a new antiferromagnetic (AFM) state, called the E-type phase, is found adjacent in parameter space to the A-type AFM phase. Its structure is in agreement with recent experimental results. This insulating E-AFM state is also competing with a ferromagnetic metallic phase as well, suggesting that large magneto-resistant effects could exist even in undoped Mn oxides. For doped layered manganites, the phase diagram includes another new AFM phase of the $\mathrm{C}_{\mathrm{x}} \mathrm{E}_{1-\mathrm{x}}$-type. Experimental signatures of the new phases are discussed.

PACS numbers: PACS numbers: 75.47.Lx, 75.30.Kz, 75.50.Ee, 75.10.-b
\end{abstract}

In the recent decade, the study of manganites - materials that show a remarkable Colossal Magneto-Resistance (CMR) 1] - has been one of the most important areas of research in condensed matter 2]. This CMR effect occurs when the manganite ground-state changes from insulating to ferromagnetic (FM) metallic after a small magnetic field is applied. Based on the concept of twophase competition [2], the CMR behavior has been successfully qualitatively reproduced in computational simulations employing resistor-network models [3]. However, more work remains to be done to fully understand Mnoxides, both regarding their unusual magneto-transport properties and the nature of the many competing phases.

The appearance of the FM metallic phase in manganites is usually rationalized by the so-called doubleexchange (DE) mechanism, based on a strong Hund coupling between mobile $e_{\mathrm{g}}$ electrons and localized $t_{2 \mathrm{~g}}$ spins. On the other hand, the insulating phase in manganites occurs due to the coupling between degenerate $e_{\mathrm{g}}$ electrons and Jahn-Teller (JT) distortions of the $\mathrm{MnO}_{6}$ octahedra, leading to the various types of charge and/or orbital orders observed experimentally [2].

The parent compound of CMR manganites is undoped $\mathrm{RMnO}_{3}$, where $\mathrm{R}$ denotes rare earth ions. For $\mathrm{R}=\mathrm{La}$, as is well-known, the A-type antiferromagnetic (AFM) phase appears, with the C-type ordering of $\left(3 x^{2}-r^{2}\right)$ and $\left(3 y^{2}-r^{2}\right)$-orbitals [4]. By substituting La by alkaline earth ions such as $\mathrm{Sr}$ and $\mathrm{Ca}$, holes are doped into the $e_{\mathrm{g}}$-electron band and due to the DE mechanism, the FM metallic phase appears, with its concomitant CMR effect. Most of the discussion in manganites has centered on the many phases induced by doping with holes the Atype AFM state, at different values of their bandwidths. In this framework, it is implicitly assumed that the undoped material is always in an A-type state. However, quite recently, a new AFM phase has been reported as the ground-state in the undoped limit for $\mathrm{R}=\mathrm{Ho}[\mathbf{5},[6]$. This phase is here called the "E-type" spin structure following the standard notation in this context. It is surprising that a new phase can be still found even in the undoped material, previously considered to be well understood. In addition, the nature of the states obtained by lightly doping this E-phase is totally unknown, and new phenomena may be unveiled experimentally in the near future. Overall, this opens an exciting new branch of investigations in manganites since novel phases appear to be hidden in the vast parameter space of these compounds. A clear example has been recently provided by the prediction of a FM charge-ordered $(\mathrm{CO})$ phase at $\mathrm{x}=1 / 2$ [7, 8], which may have been found experimentally already $[9]$.

In this Letter, based on the orbital-degenerate DE model coupled with JT distortions, the ground state properties of undoped manganites are analyzed by using mean-field (MF) calculations and Monte-Carlo (MC) simulations. In our phase diagram at $\mathrm{x}=0$, the $\mathrm{E}-\mathrm{AFM}$ phase is found to exist in a wide region of parameter space, adjacent to the A-AFM phase in agreement with the experimental results. The E-AFM phase is robust when the dimensionality and/or electron-phonon coupling are modified, and its strength originates in the intrinsic nature of orbital-degenerate DE systems. In the phase diagram and at small electron-phonon coupling the E-AFM insulating phase is located just next to a FM metallic phase 10, suggesting that CMR effects could be found even at $\mathrm{x}=0$. Light-hole doping $\mathrm{x}$ of the E-type phase is also discussed and another novel magnetic phase, defined as the " $\mathrm{C}_{\mathrm{x}} \mathrm{E}_{1-\mathrm{x}}$ " phase, is found. The ubiquitous phase-separation tendencies observed when insulating and metallic phases compete is also expected near the FM- $\mathrm{C}_{\mathrm{x}} \mathrm{E}_{1-\mathrm{x}}$ boundary.

The Hamiltonian studied in this paper is

$$
\begin{aligned}
H & =-\sum_{\mathbf{i} \mathbf{a} \gamma \gamma^{\prime} \sigma} t_{\gamma \gamma^{\prime}}^{\mathbf{a}} d_{\mathbf{i} \gamma \sigma}^{\dagger} d_{\mathbf{i}+\mathbf{a} \gamma^{\prime} \sigma}-J_{\mathrm{H}} \sum_{\mathbf{i}} \mathbf{s}_{\mathbf{i}} \cdot \mathbf{S}_{\mathbf{j}} \\
& +J_{\mathrm{AF}} \sum_{\langle\mathbf{i}, \mathbf{j}\rangle} \mathbf{S}_{\mathbf{i}} \cdot \mathbf{S}_{\mathbf{j}}+\lambda \sum_{\mathbf{i}}\left(Q_{1 \mathbf{i}} \rho_{\mathbf{i}}+Q_{2 \mathbf{i}} \tau_{\mathbf{x i}}+Q_{3 \mathbf{i}} \tau_{\mathbf{z i}}\right) \\
& +(1 / 2) \sum_{\mathbf{i}}\left(\beta Q_{1 \mathbf{i}}^{2}+Q_{2 \mathbf{i}}^{2}+Q_{3 \mathbf{i}}^{2}\right)
\end{aligned}
$$


where $d_{\mathbf{i a} \sigma}\left(d_{\mathbf{i b} \sigma}\right)$ annihilates an $e_{\mathrm{g}}$-electron with spin $\sigma$ in the $d_{x^{2}-y^{2}}\left(d_{3 z^{2}-r^{2}}\right)$ orbital at site $\mathbf{i}$, and $\mathbf{a}$ is the vector connecting nearest-neighbor (NN) sites. The first term is the NN hopping of $e_{\mathrm{g}}$ electrons with amplitude $t_{\gamma \gamma^{\prime}}^{\mathrm{a}}$ between $\gamma$ - and $\gamma^{\prime}$-orbitals along the a-direction: $\quad t_{\mathrm{aa}}^{\mathbf{x}}=-\sqrt{3} t_{\mathrm{ab}}^{\mathbf{x}}=-\sqrt{3} t_{\mathrm{ba}}^{\mathbf{x}}=3 t_{\mathrm{bb}}^{\mathbf{x}}=t$ for $\mathbf{a}=\mathbf{x}$, $t_{\mathrm{aa}}^{\mathbf{y}}=\sqrt{3} t_{\mathrm{ab}}^{\mathbf{y}}=\sqrt{3} t_{\mathrm{ba}}^{\mathbf{y}}=3 t_{\mathrm{bb}}^{\mathbf{y}}=t$ for $\mathbf{a}=\mathbf{y}$, and $t_{\mathrm{bb}}^{\mathbf{z}}=4 t / 3$ with $t_{\mathrm{aa}}^{\mathbf{z}}=t_{\mathrm{ab}}^{\mathbf{z}}=t_{\mathrm{ba}}^{\mathbf{z}}=0$ for $\mathbf{a}=\mathbf{z}$. Hereafter, $t$ is taken as the energy unit. In the second term, the Hund coupling $J_{\mathrm{H}}(>0)$ links $e_{\mathrm{g}}$ electrons with spin $\mathbf{s}_{\mathbf{i}}=\sum_{\gamma \alpha \beta} d_{\mathbf{i} \gamma \alpha}^{\dagger} \boldsymbol{\sigma}_{\alpha \beta} d_{\mathbf{i} \gamma \beta}$ $\left(\boldsymbol{\sigma}=\right.$ Pauli matrices) with the localized $t_{2 \mathrm{~g}}$ spin $\mathbf{S}_{\mathbf{i}}$ assumed classical with $\left|\mathbf{S}_{\mathbf{i}}\right|=1 . \quad J_{\mathrm{H}}$ is here considered as infinite or very large. The third term is the AFM coupling $J_{\mathrm{AF}}$ between $\mathrm{NN} t_{2 \mathrm{~g}}$ spins. The fourth term couples $e_{\mathrm{g}}$ electrons and $\mathrm{MnO}_{6}$ octahedra distortions [1], $\lambda$ is a dimensionless coupling constant, $Q_{1 \mathbf{i}}$ is the breathing-mode distortion, $Q_{2 \mathbf{i}}$ and $Q_{3 \mathbf{i}}$ are, respectively, $\left(x^{2}-y^{2}\right)$ - and $\left(3 z^{2}-r^{2}\right)$-type JT-mode distortions, $\rho_{\mathbf{i}}=$ $\sum_{\gamma, \sigma} d_{\mathbf{i} \gamma \sigma}^{\dagger} d_{\mathbf{i} \gamma \sigma}, \tau_{\mathrm{xi}}=\sum_{\sigma}\left(d_{\mathbf{i} a \sigma}^{\dagger} d_{\mathbf{i b} \sigma}+d_{\mathbf{i b} \sigma}^{\dagger} d_{\mathbf{i} a \sigma}\right)$, and $\tau_{\mathrm{zi}}=$ $\sum_{\sigma}\left(d_{\mathbf{i} a \sigma}^{\dagger} d_{\mathbf{i} a \sigma}-d_{\mathbf{i} b \sigma}^{\dagger} d_{\mathbf{i} b \sigma}\right)$. The fifth term is the usual quadratic potential for adiabatic distortions and $\beta$ is the spring-constants ratio for breathing- and JT-modes. In actual manganites, $\beta \approx 2$. In undoped manganites, all oxygens are shared by adjacent $\mathrm{MnO}_{6}$ octahedra and the distortions are not independent, suggesting that the cooperative effect is even more important than for the doped case $x>0$. To consider this cooperation, here oxygen ion displacements are directly optimized [12].

Let us first describe our two-dimensional (2D) results, since the essential physics behind the stabilization of the E-type phase can be grasped by using MC simulations with relatively short CPU times. The phase diagram on a $4 \times 4$ lattice is shown in Fig. 1(a). The continuous curves are obtained by comparing the energies of the competing phases in the MF calculations, while the circles are obtained by monitoring the nature of the dominant spin correlation $S(\mathbf{q})$ in MC simulations. A typical result for $S(\mathbf{q})$ is shown in Fig. 1(b). A new regime characterized by $\mathbf{q}=(\pi / 2, \pi / 2)$ is clearly observed between the FM and G-AFM phases. The good agreement between MF and $\mathrm{MC}$ results in the region of interest shows the high accuracy of the present MF calculations for manganites [14].

In Fig. 1(c), the spin and orbital structure of the novel intermediate phase (E-phase) is shown. Along the zigzag chains, $t_{2 \mathrm{~g}}$ spins order ferromagnetically, but they are antiparallel perpendicular to the zigzag direction. In the three-dimensional (3D) case, the MF study shows that the pattern Fig. 1(c) just stacks along the $z$-axis, while the spin directions are reversed from plane to plane. Note that the orbital structure is the same as that of the AAFM phase, namely, the staggered pattern of $\left(3 x^{2}-r^{2}\right)$ and $\left(3 y^{2}-r^{2}\right)$-like orbitals. Our investigations show that the E-phase is robust at weak- and intermediate- $\lambda$, but for $\lambda>1.5$, the E-type regime narrows.

A surprising aspect of our results is that the E-type spin arrangement is the ground-state for a wide range of $J_{\mathrm{AF}}$, even at $\lambda=0$, indicating that the coupling with
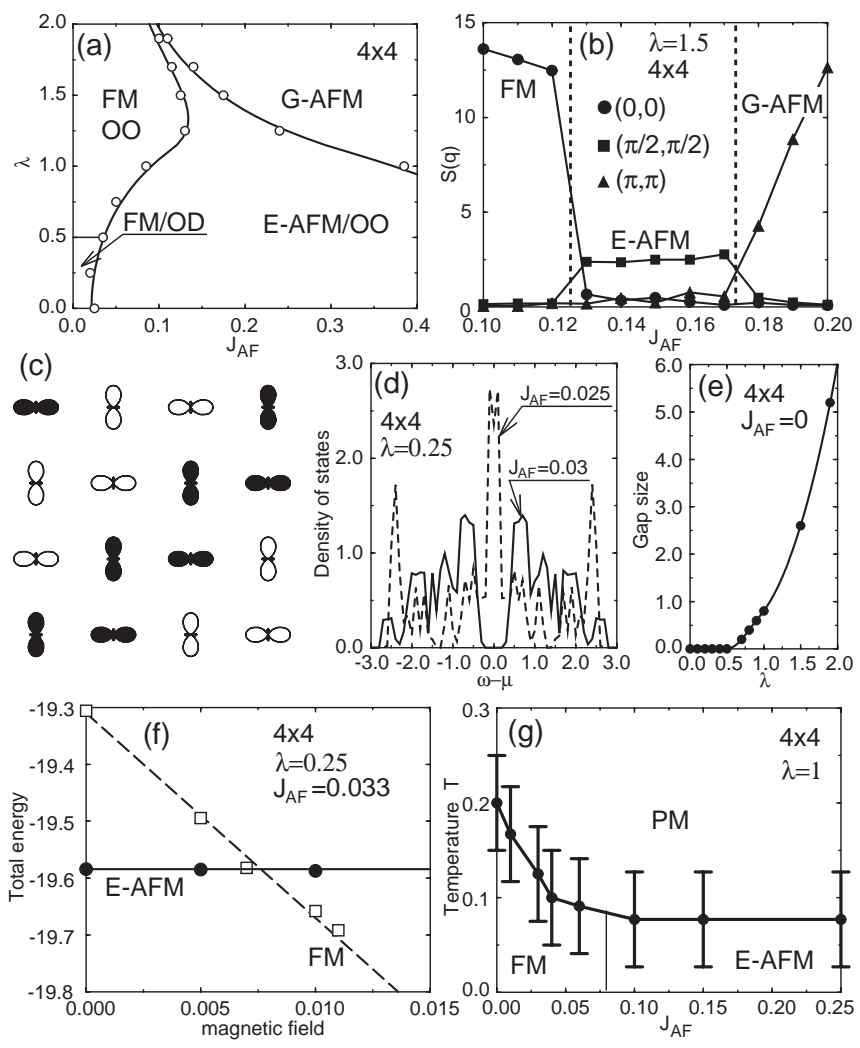

FIG. 1: (a) Phase diagram obtained using the $4 \times 4$ lattice. Open circles denote MC results and solid lines are obtained with MF calculations. (b) Spin correlation $S(\mathbf{q})$ vs. $J_{\mathrm{AF}}$ at $\lambda=1.5$. (c) Schematic view of the spin and orbital structure of the E-AFM phase. Solid and open symbols denote orbitals with up- and down-spins, respectively. Note the presence of zigzag chains for each spin orientation. (d) Density of states (DOS) obtained by MC simulations at $\lambda=0.25$. Solid and dashed curves denote the results for E- and FM-phases. (e) Gap at the Fermi level for the FM phase vs. $\lambda$. The magnitude is evaluated from the DOS obtained by MC simulations. (f) Total energy vs. magnetic field for E-AFM and FM phases. (g) Phase diagram in the $\left(J_{\mathrm{AF}}, T\right)$ plane at $\mathrm{x}=0$ and $\lambda=1$, using a $4 \times 4$ lattice. Estimations of the Curie and Néel temperatures are evaluated using MC simulations (see text).

JT phonons is not a necessary condition for its stabilization. This is in sharp contrast to the case of the A-AFM phase. To understand this point, it is instructive to study the $e_{\mathrm{g}}$-electronic structure of the zigzag FM chains that appear in the E-phase spin arrangement. Taking $J_{\mathrm{H}}$ as infinity for simplicity, the $e_{\mathrm{g}}$ electrons move only along the zigzag FM chain, and cannot hop to the adjacent FM chains. The dispersion energy for $e_{\mathrm{g}}$ electrons in this zigzag FM chain is given by $\varepsilon_{k}=(2 / 3)\left(\cos k \pm \sqrt{\cos ^{2} k+3}\right)$ and $(2 / 3)\left(-\cos k \pm \sqrt{\cos ^{2} k+3}\right)$, indicating that there appears a large band-gap equal to $4 t / 3$ at half-filling. In fact, even on the $4 \times 4$ cluster, there is a clear gap of the order of $t$ for the E-phase in the density of states (DOS) (Fig. $1(\mathrm{~d}))$. Since $t_{\mu \nu}^{\mathbf{x}}=-t_{\mu \nu}^{\mathbf{y}}$ for $\mu \neq \nu$, the sign in the hopping amplitude changes periodically in the zigzag situa- 

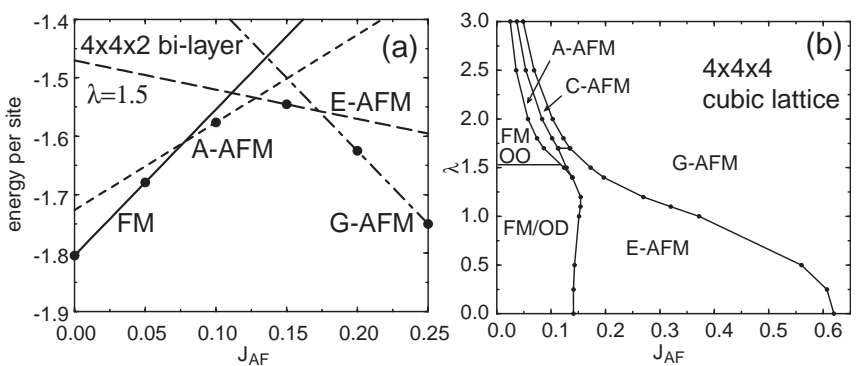

FIG. 2: (a) Energies of the FM, A-AFM, E-AFM, C-AFM, and G-AFM phases on $4 \times 4 \times 2$ bi-layer lattices $(\lambda=1.5)$. Solid circles indicate the results of optimizations, while lines denote the MF results. (b) Phase diagram for the $4 \times 4 \times 4$ cubic lattice. All solid lines emerge from MF calculations.

tion, leading to a periodic potential for $e_{\mathrm{g}}$ electrons and its concomitant band-insulator nature. In other words, the E-type phase is stable due to the zigzag geometry of the FM chains that induce a band-insulator [15].

Another related interesting point is the orbital structure of the FM phase. In the strong-coupling region, an orbitally ordered (OO) state appears, essentially with the same pattern as that observed in the E-AFM phase discussed above. All $\mathrm{MnO}_{6}$ octahedra are distorted at $\mathrm{x}=0$ and the cooperative effect is essential to determine the OO pattern for large $\lambda$, irrespective of the spin structure. However, when $\lambda$ decreases OO disappears and an orbital disordered (OD) new phase is observed. This is a metallic phase according to the DOS in Fig. 1(d). The OO-OD transition is monitored by the gap size at the Fermi level in the DOS (Fig. 1(e)). The transition observed using a $4 \times 4$ cluster is robust, although the actual critical value may change in larger systems 16 .

Note that the OD/FM phase is next to the insulating E-AFM phase for $\lambda \lesssim 0.5$ (the E-phase is insulating with a DOS gap both at small and large $\lambda$ ). Such a result is new in the study of undoped manganites. Since the competition between FM metallic and insulating phases is at the heart of the CMR phenomena, by tuning experimentally the lattice parameters in $\mathrm{RMnO}_{3}$ it may be possible to observe the magnetic-field induced metal-insulator transition even in undoped manganites. In fact, small magnetic fields of a few Teslas indeed induce a phase transition between E- and FM phases (see Fig. 1(f)), with a concomitant jump in the magnetization.

Let us discuss the effect of thermal fluctuations. For this purpose, both $T_{\mathrm{C}}$ (Curie temperature) and $T_{\mathrm{N}}$ (Néel temperature) are estimated from MC simulations in $4 \times 4$ clusters 17] (Fig. 1(g)). Our estimated $T_{\mathrm{C}}$ is found to decrease with increasing $J_{\mathrm{AF}}$, and in the E-AFM phase, $T_{\mathrm{N}}$ becomes almost constant. The E-phase can be disordered by thermal fluctuations faster upon heating than the FM phase. Provided that our estimated $T_{\mathrm{C}}$ in $2 \mathrm{D}$ system is related to $T_{\mathrm{N}}$ with the A-AFM phase of $3 \mathrm{D}$ lattices, Fig. 1(g) mimics well the experimental results for the changes of $T_{\mathrm{N}}$ by R-ion substitution in $\mathrm{RMnO}_{3}[6]$.

Now let us turn our attention to the effect of dimen-
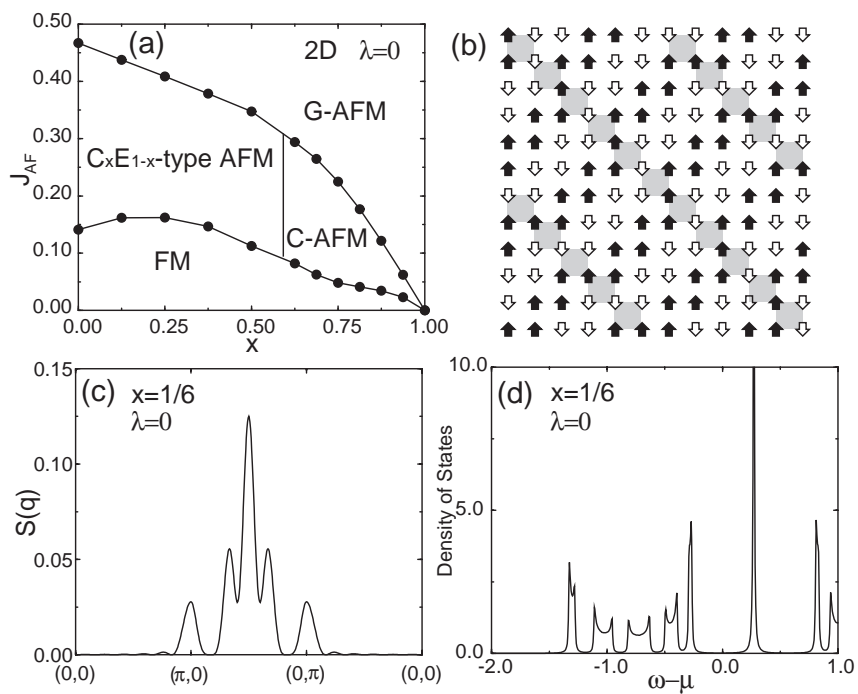

FIG. 3: (a) Phase diagram in the $\left(\mathrm{x}, J_{\mathrm{AF}}\right)$ plane for layered manganites at $\lambda=0$ obtained by analytic calculations. (b) Schematic view for spin structure of the $\mathrm{C}_{\mathrm{x}} \mathrm{E}_{1-\mathrm{x}}$-type phase at $\mathrm{x}=1 / 6$. Hatched squares denote hole-rich C-type regions. (c) $S(\mathbf{q})$ and (d) the DOS in the $\mathrm{C}_{1 / 6} \mathrm{E}_{5 / 6}$-type phase.

sionality. In Fig. $2(\mathrm{a})$, using bi-layer $4 \times 4 \times 2$ clusters, the ground-state energy per site is depicted vs. $J_{\mathrm{AF}}$ at $\lambda=1.5$ both for numerical optimization [18] and MF calculations, with a good agreement between them. Figure 2(b) is the phase diagram on a $4 \times 4 \times 4$ cubic lattice, obtained only by the MF approximation, since MC calculations in this case are too CPU time consuming (previous MF$\mathrm{MC}$ comparisons suggest that this MF phase diagram is accurate). In the strong-coupling region, there occurs a chain of transitions from $\mathrm{FM} \rightarrow \mathrm{A}-\mathrm{AFM} \rightarrow \mathrm{C}-\mathrm{AFM} \rightarrow \mathrm{G}-$ AFM phases 19], already obtained in $2 \times 2 \times 2$ calculations [4]. The present result shows that size effects are small in undoped strongly-coupled manganites, which is intuitively reasonable. Note that near $\lambda \sim 1.6$, a realistic value for manganites, the A-AFM phase is adjacent to the Etype state. This region could correspond to the actual situation observed in experiments for $\mathrm{RMnO}_{3}$ : When the ionic radius of the R-site decreases, $T_{\mathrm{N}}$ of the A-AFM phase decreases as well, and eventually the E-AFM phase is stabilized for $\mathrm{R}=\mathrm{Ho}[\underline{6}$. In the weak-coupling region, the E-type phase is stable in a wide range of $J_{\mathrm{AF}}$, as in the $2 \mathrm{D}$ calculation.

Consider now the very interesting effect of light hole doping on the E-phase. Hole doping will be here studied in the weak coupling limit, since the E-type phase is well understood at $\lambda=0$. Figure 3(a) shows the groundstate phase diagram in the $\left(\mathrm{x}, J_{\mathrm{AF}}\right)$ plane, obtained using analytic calculations on $2 \mathrm{D}$ lattices at $\lambda=0$. A remarkable feature of this phase diagram is the appearance of the novel $\mathrm{C}_{\mathrm{x}} \mathrm{E}_{1-\mathrm{x}}$ phase, composed of long-period zigzag FM chains, antiferromagnetically coupled to each other (see Fig. 3(b) for $x=1 / 6$ ). As the doping fraction grows, it is expected that the previously reported 
states at intermediate- and high-doping will eventually be reached 8,13

Note that the curvature of the boundary between FM and $\mathrm{C}_{\mathrm{x}} \mathrm{E}_{1-\mathrm{x}}$ phases is found to be negative for $0 \lesssim \mathrm{x} \lesssim 0.5$, indicating that phase separation occurs between those two phases. Here it is again stressed that the FM phase is metallic, while $\mathrm{C}_{\mathrm{x}} \mathrm{E}_{1-\mathrm{x}}$ is insulating. The latter can be considered as a microscopic phase separated state, since the C- and E-type structure are mixed at the length scale of a lattice constant. This phase is expected to be made unstable easily near the phase boundary region, and to turn into a phase-separated state with a mixture of metallic FM and insulating $\mathrm{C}_{\mathrm{x}} \mathrm{E}_{1-\mathrm{x}}$ clusters, which may induce CMR effects.

In the $\mathrm{C}_{\mathrm{x}} \mathrm{E}_{1-\mathrm{x}}$ phase, $e_{\mathrm{g}}$ holes tend to localize in the C-type region (hatched squares in Fig. 3(b)), i.e., in the straight segment portion of the zigzag FM chain, and as a consequence charge-ordering of the stripe form is induced even in the $\lambda=0$ limit [20]. This causes incommensurate peaks in the charge correlation at $\mathbf{q}=(2 \pi \mathrm{x}, 2 \pi \mathrm{x})$. The spin sector is also nontrivial (Fig. 3(c)) and the state is an insulator according to the DOS (Fig. 3(d)) 21].

In summary, the extended phase diagrams of mangan- ites for $\mathrm{x}=0$ and $\mathrm{x}>0$ have been discussed. A novel EAFM phase, stabilized at $\mathrm{x}=0$ in the region of weak- and intermediate-couplings, is adjacent to both FM metallic and A-AFM states. The competition between E-AFM insulating and FM metallic phases suggests the possibility of CMR effects even in undoped manganites. Several features of the E- to A-AFM transition, at least at the qualitative level, agrees with currently available experimental results. For the doped case, a microscopically inhomogeneous $\mathrm{C}_{\mathrm{x}} \mathrm{E}_{1-\mathrm{x}}-\mathrm{AFM}$ state is predicted. This state may contribute to the phase separation tendencies widely observed experimentally in Mn-oxides for $0 \lesssim \mathrm{x} \lesssim 0.5$. The discovery of these many "hidden" interesting phases in real manganites - undoped and doped - should be actively pursued experimentally. This will open a new subbranch of investigations in the already much interesting context of Mn oxides.

The authors thank T. Kimura and K. Ueda for discussions. T.H. has been supported by a Grant-in-Aid for Scientific Research from the Ministry of Education, Culture, Sports, Science, and Technology of Japan. E. D. is supported by the NSF grant DMR-0122523.
[1] See, for instance, Colossal Magnetoresistance Oxides, edited by Y. Tokura, Gordan \& Breach, New York, 2000.

[2] E. Dagotto et al., Phys. Rep. 344, 1 (2001). See also E. Dagotto, Nanoscale Phase Separation and Colossal Magnetoresistance, Springer-Verlag, Berlin, 2002.

[3] M. Mayr et al., Phys. Rev. Lett. 86, 135 (2001); J. Burgy et al., Phys. Rev. Lett. 87, 277202 (2001).

[4] T. Mizokawa and A. Fujimori, Phys. Rev. B54, 5368 (1995); W. Koshibae et al., J. Phys. Soc. Jpn. 66, 957 (1997); S. Ishihara et al., Phys. Rev. B55, 8280 (1997); R. Maezono et al., Phys. Rev. B57, R13993 (1998); L. F. Feiner and A. M. Oleś, Phys. Rev. B59, 3295 (1999); P. Horsch et al., Phys. Rev. B59, 6217 (1999); J. van den Brink et al., Phys. Rev. B59, 6795 (1999); T. Hotta et al., Phys. Rev. B60, R15009 (1999); M. Capone et al., Euro. Phys. J. B17, 103 (2000).

[5] A. Muñoz et al., Inorg. Chem. 40, 1020 (2001).

[6] T. Kimura et al., cond-mat/0211568

[7] S. Yunoki et al., Phys. Rev. Lett. 84, 3714 (2000).

[8] T. Hotta et al., Phys. Rev. Lett. 84, 2477 (2000).

[9] J. Loudon et al., Nature 420, 797 (2002).

[10] Although $\mathrm{x}=0$ corresponds to one $e_{g}$ electron per site which naively may suggest insulating behavior, there are two active orbitals and the mean density per state is $1 / 2$. Note also that previous work 2] has shown that a large Hund coupling is equivalent to a large Hubbard repulsion.

[11] Since JT distortions suppress double occupancy as shortrange Coulomb interaction does, a large $\lambda$ is quite fine to mimic the physics of manganites, as shown in [2].

[12] In practice, considering sites $\mathbf{i}$ and $\mathbf{i}+\mathbf{a}$, the oxygen in between is allowed to move along the a-axis, i.e., buckling and rotations are neglected. In the $2 \mathrm{D}$ case apical oxygens are assumed to be fixed, consistent with previous treatments to produce stripe-like charge ordering in the 2D FM-phase [13].
[13] T. Hotta et al., Phys. Rev. Lett. 86, 4922 (2001).

[14] T. Hotta et al., Phys. Rev. B62, 9432 (2000).

[15] A similar mechanism has been discussed by some of the authors for the CE-type phase at $\mathrm{x}=0.5[8]$.

[16] By analytic calculations it has been confirmed that the metal-insulator transition driven by JT phonons occurs at a finite value of $\lambda$ in the infinite lattice. (see T. Hotta, cond-mat/0212575).

[17] While finite clusters do not show true thermodynamic singularities, a rapid increase in the strength of correlations is observed at fairly well defined temperatures, here referred to as $T_{\mathrm{N}}$ and $T_{\mathrm{C}}$ for simplicity. Previous computational experience shows that these estimations are fairly accurate upon increasing lattice sizes [2].

[18] Both spin directions and oxygen positions are optimized.

[19] Note that the FM/OO state of Fig. 2(b) is another phase not previously observed experimentally, here predicted to exist for low- $J_{\mathrm{AF}}$ manganites at $\mathrm{x}=0$.

[20] As in any striped state, further work should be carried out to check the stability of the structure against longrange Coulomb interactions.

[21] For $\mathrm{x}>0.5$ in the strong-coupling case, the $\mathrm{C}_{\mathrm{x}} \mathrm{E}_{1-\mathrm{x}^{-}}$ phase is compatible with the so-called Wigner-crystal $\mathrm{CO} / \mathrm{OO}$ structure suggested for $\mathrm{x}=2 / 3$ and $3 / 4$ in $\mathrm{La}_{1-\mathrm{x}} \mathrm{Ca}_{\mathrm{x}} \mathrm{MnO}_{3}$ (P. G. Radaelli et al., Phys. Rev. B59, 14440 (1999); M. T. Fernández-Díaz et al., Phys. Rev. B59, 1277 (1999)). However, the bi-stripe CO/OO phase suggested by Mori et al. (S. Mori et al., Nature 392, $473(1998))$ is consistent with the $\mathrm{C}_{1-\mathrm{x}} \mathrm{E}_{\mathrm{x}}-\mathrm{AFM}$ state, which emerges from the competition with the Wignercrystal structure [8]. Experimentally, the $\mathrm{C}_{\mathrm{x}} \mathrm{E}_{1-\mathrm{x}}-\mathrm{AFM}$ phase has been reported for $\mathrm{x}>0.5$ in $\mathrm{Nd}_{1-\mathrm{x}} \mathrm{Sr}_{1+\mathrm{x}} \mathrm{MnO}_{4}$ (T. Kimura et al, Phys. Rev. B65, 020407(R) (2001)). 